\title{
Recent patents in microfabrication and microfluidics
}

\begin{abstract}
Patent \# Subject
WO 9904911 Formation of silicon film on structure surfaces by exposing the structure to an electrically neutral reactive gaseous phase and maintaining it at a temperature below that of the reactive gaseous phase; used to form a silicon film on wire, neural probes, microfabrication substrates, silicon wafers, implantable biomedical devices, or neurological electrode assemblies.

WO 9903584 Disposable microfluidic analyzer for measuring glucose, lactate ammonium phosphate, iron in whole or diluted blood, plasma, serum, etc.; comprises channels defined at the interface of two superposed polymer layers directly bonded together without adhesive.

WO 9902266 Assay device comprising a metal oxide membrane substrate with permeating channels that form a sample application zone carrying a specific binding agent, particularly for detecting human immunodeficiency virus.

US 5852495 Electrophoretic analysis method involving the illumination of a channel at regularly spaced regions and detecting fluorescent emissions from separated species of materials passing through the channel; used for the analysis of chemical compounds, polysaccharides, small organic and inorganic molecules, and biological macromolecules.
\end{abstract}

WO 9856956 Determination of the rate of reactions in microfluidic systems by measuring velocities of components reacting to form product and concentration of product, and calculating the rate of reaction.

WO 9856505 In situ dilution or concentration of materials in a microfluidic system handling sufficiently small volumes of fluid, so that multiple operations can be integrated into a single low-volume device and performed automatically and with a high degree of precision.

WO 9855852 Introduction of fluids into a device with microfluidic channels, with fluids formed as drops on outer surfaces of arrays of pins and wicked into channels by capillary forces; useful for the analysis of chemical and biological specimens.

US 5842787 Microfluidic device for ultrahigh-throughput screening assay system (e.g., for pharmaceutical development, diagnostics, immunoassays, and nucleic acid analysis); has a microscale channel with a nonmixing region, and a mixing region with sufficient cross-sectional area to permit diffusional mixing.

WO 9853311 Apparatus that drives fluid through a microfluidic system using centripetal force-has a platform rotatable at various speeds to manipulate the flow of fluids to different chambers; for performing microanalysis, synthesis, and purification in genetic, biochemical, and chemical fields.

WO 9850773 Microcantilever biosensor for molecular diagnostics of diseaseshas a cantilever microbeam that responds to chemical stimulus by producing an electrical output (e.g., resonant frequency), resulting in a lower-cost and less labor-intensive sensor.

WO 9849548 Microfluidic device used for the analysis of multiple sampleshas interconnecting microscale channels connected to sample and waste reservoirs, permitting faster analysis of multiple samples with minimal cost, space, and time.

WO 9846438 A microfluidic system for chemical and biochemical analysis, synthesis, and detection, comprising a polymeric body having intersecting channels with zeta potential-associated interior surfaces, electrical ports, and an electrical control system.

WO 9845481 Nucleic acid sequencing using primer, nucleotide, and polymerase, and carried out in a microfluidic system (e.g., in a new microfluidic apparatus), useful for amplification studies or immunoassays; particularly used for the complete sequencing of (large) DNA molecules by iterative sequencing of sub-sequences, without needing to synthesize new primers.

$\begin{array}{llll}\text { Assignee } & \text { Author } & \text { Date } & \text { Status } \\ \begin{array}{lll}\text { MIT } \\ \text { (Cambridge, MA) }\end{array} & \text { Gleason KK, Kwan MC } & \text { 2/4/99 } & \text { A1 }\end{array}$

YSI Inc.

Moles DR

$1 / 28 / 99$ A1

(Yellow Springs, $\mathrm{OH}$ )

Kreuwel HJM,

$1 / 21 / 99 \quad A 1$

AKZO Nobel NV

(Arnhem,

van Damme HS

$12 / 2 / 98 \quad A$

Caliper Technologies Parce JW

(Palo Alto, CA)

$\begin{array}{ll}\text { Caliper Technologies } & \text { Chow AW, Cohen CB, } \\ \text { (Palo Alto, CA) } & \text { Kopf-Sill AR, Parce JW, }\end{array}$

Sundberg SA

Caliper Technologies Kopf-Sill AR, Parce JW 12/17/98 A1

(Palo Alto, CA)

Caliper Technologies Chow CYH, Parce JW, 12/10/98 A1 (Palo Alto, CA) Sundberg $\mathrm{S}$

Caliper Technologies Kopf-Sill AR, Parce JW 12/10/98 A (Palo Alto, CA)

Gamera Bioscience

(Medford, MA)

Univ. Minnesota

(Minneapolis, MN)

Caliper Technologies (Palo Alto, CA)

Caliper Technologies Bousse L, Chow CYH, (Palo Alto, CA) Knapp MR, Parce JW

Caliper Technologies Bousse LJ, Knapp M, (Palo Alto, CA)
Carvalho BL, Chapman 11/26/98 A2 LL, Davis GA, Kellogg G, Kieffer-Higgins SG, Kob $\mathrm{M}$, Mian A, Minior T, Oeltjen $\mathrm{SD}$, Ommert S, Willis JP

Charych DH, McGlennen RC,

$11 / 12 / 98$ A2 Polla DL

Bousse L, Dubrow RS， 11/5/98 A1 Kennedy CB
$10 / 22 / 98 \mathrm{~A} 1$ 10/15/98 A1 